\title{
Prácticas universitarias: una perspectiva integral
}

\section{Universitary practices: an integrated perspective}

Karina Oldani

https://orcid.org/0000-0002-3685-8725

karinaoldani@gmail.com

Facultad de Ciencias Naturales

y Museo | UNLP | Argentina

\author{
Martina Cecotti \\ https://orcid.org/0000-0002-6726-4946 \\ cecotti@quimica.unlp.edu.ar \\ Facultad de Ciencias Exactas | \\ UNLP I
}

Leandra Hanlon

https://orcid.org/0000-0003-0219-9247

arqlhanlon@gmail.com

Facultad de Arquitectura

y Urbanismo | UNLP | Argentina

\section{RESUMEN}

A partir de la experiencia transitada en el Seminario de la Especialización en Docencia Universitaria "Políticas de articulación, docencia, investigación, extensión y transferencia", reflexionamos acerca de obstáculos o límites que se presentan en el camino hacia una dinámica de formación con perspectiva integral en nuestras universidades. Proponemos un Taller donde los conocimientos prácticos, académicos y extra-académicos puedan integrarse en el análisis y búsqueda de soluciones a problemas reales. Este espacio sería posible de ser incorporado en el currículum de cualquier carrera universitaria, encuadrado en aprendizaje basado en problemas (ABP), donde los estudiantes se enfrentan al problema antes de un determinado aprendizaje: es decir, el problema guía al aprendizaje. Imaginamos un contraste con aquella situación inicial desde la cual los imaginarios o supuestos acerca de las capacidades individuales excluyentes o incumbencias exclusivas de cada campo disciplinar, podría redefinirse y orientarse hacia una visión más amplia e integradora de los saberes específicos, y contribuir con un aprendizaje cooperativo o cooperado que refuerce su vínculo de pertenencia tanto con la comunidad académica de un sistema educativo efectivamente público y democrático, como con la sociedad con la cual interactúen solidariamente, en pos de una sociedad plural y democrática.

PALABRAS CLAVE

interdisciplinariedad, articulación, compromiso social, integralidad 


\section{KEY WORDS}

interdisciplinarity articulation,

social commitment

integral knowledge

\section{ABSTRACT}

Based on the experience in the Especialización en Docencia Universitaria Seminar "Políticas de articulación, docencia, investigación, extensión y transferencia", we reflect about obstacles or limitations that arise along the pathway to an education modality with an integrated perspective in the University. We propose a workshop where practical, academic and extra-academic knowledge could be integrated in order to study and reach solutions to real-life problems. The workshop could be incorporated in the curriculum of any university career. A learning space designed within Problem Based Learning (PBL), where students face a problem to be solved before knowing the theory: working out the solutions leads the learning experience. We believe in the turning of the social imaginarium or assumptions about exclusive bodies of knowledge to a complementary vision of the different specific disciplines, redefining the idea of boundaries towards an integrated and broader perspective. This could contribute to the development of cooperative learning in order to strengthen the students' bond both with an academic community within a genuinely public and democratic educational system, and with the society they would be interacting in, in pursuit of a plural and more democratic society. 


\section{PRESENTACIÓN: REFLEXIÓN Y DIAGNÓSTICO}

A partir de la experiencia transitada en el Seminario de la Especialización en Docencia Universitaria "Políticas de articulación, docencia, investigación, extensión y transferencia", podemos recapitular una serie de reflexiones que son identificables como obstáculos o límites en el camino hacia una dinámica de formación con perspectiva integral. Sin embargo, creemos que son estos mismos los temas de trabajo con mayor potencial a la hora de pensar estrategias de enseñanza y aprendizaje superadoras, no sólo de aquellas que actualmente predominan en los ámbitos académicos y que mayormente replicamos en el ámbito de la docencia, sino incluso de las que fueron los moldes de nuestra propia trayectoria formativa.

Desde esta visión inicial consideramos:

- Que dicha formación se caracteriza por la fragmentación de conocimientos, por una visión de la educación como acumulación de los mismos y una formación individualista, tendiente a generar capital humano especializado en un modelo de universidad que es presionado por las demandas del neoliberalismo (Coraggio, 2015; Jara Holliday, 2018; Rafaghelli, 2013). La formación de estos profesionales especialistas se limita a la resolución de problemas disciplinares específicos, o incluso a parcialidades de esos problemas, evidenciando una falta de interdisciplinariedad y de cooperación institucional que es necesario superar para abordar las complejas problemáticas de la realidad social (Coraggio, 2015).

- Que existe una tensión entre un modelo de Universidad que propone prácticas educativas tendientes a la formación de sujetos de transformación, capaces de comprender su potencial en el contexto del cual forman parte, construyendo ciudadanías democráticas, comprometidos con la sociedad, y un modelo que responde al neoliberalismo, pretendiendo una "Universidad del Cálculo", negando su compromiso con las demandas/necesidades de la comunidad (Müller, 1996, como se citó en Sutz, 2010:48). 
- Que esta situación puede asociarse con la fragmentación que se replica en sucesivos espacios dentro de la Universidad. Por un lado, las acciones del Consejo Social (CS) buscan recuperar/construir un circuito de preguntas recíprocas, a la vez que articula las funciones básicas de la Universidad y los diversos actores sociales en la co-construcción de demandas/problemas, sin la suficiente difusión de esta tarea. Por el otro, vemos que en las diversas Unidades Académicas coexisten esfuerzos aislados en proyectos de extensión que podrían conectarse e integrarse entre sí pero que se desarrollan por separado, y hasta con desconocimiento de la existencia del CS.

Desde el ámbito académico, existe coincidencia en la lectura de los puntos anteriores y una genuina preocupación por promover la construcción de proyectos desde otras lógicas. Desde el ámbito profesional, compartimos la condición fundamental de encontrarnos del otro lado de "la meta", donde esperábamos cumplida la promesa del sistema de "estar capacitados" para la vida laboral, y en cambio nos topamos con situaciones "reales" donde los colegas adentrados en la práctica nos recibieron ávidos de contarnos todo eso "para lo que no te forma la facultad".

Desde esta experiencia, buscamos reformular las propias estrategias de enseñanza-aprendizaje, pero contamos con herramientas que continúan siendo fragmentarias, parciales y asistemáticas. Los vicios de nuestra propia estructura de formación, enmarcados en el "habitus académico" (Ezcurra, 2011:145), tienden a definir nuestras acciones sobre componentes parciales del proceso educativo. El desafío es poder concretar una propuesta de integralidad partiendo de la contextualización de las prácticas universitarias e incorporando la problematización como práctica verdaderamente transversal.

\section{El desafío es poder concretar una propuesta de integralidad partiendo de la contextualización de las prácticas universita- rias e incorporando la problematización como práctica verda- deramente transversal.}

\section{PROBLEMATIZACIÓN DEL CONTEXTO: PROPUESTA Y ESTRATEGIAS RESPECTO A LOS ASPECTOS INVOLUCRADOS}

Retomando lo expuesto por González Valdés (2001) sobre la concentración de esfuerzos en los niveles terminales de la educación (por la propia demanda del sistema educacional sobre sí mismo, sea en sus bases o sobre la acumulación de saldos pendientes), el momento de nuestra propuesta de intervención sería el inicio del recorrido universitario, permitiendo a los estudiantes conocer, desde el ingreso, su potencial de acción en la problematización y posible solución de demandas ac- 
tuales de la sociedad. Creemos oportuna esta construcción en el punto de partida, buscando una propuesta que abra una puerta a la Universidad, tras la cual vean otras puertas por abrir, oponiéndose al modelo de "puerta giratoria" (Ezcurra, 2011:133).

Con base a lo expuesto encontramos tres puntos de partida complementarios para la acción:

- Focalizar en los espacios de ARTICULACIÓN, desde las políticas institucionales de articulación de áreas (como la conformación de organismos de co-gestión como el CS) a la promoción desde cada disciplina del trabajo colaborativo interdisciplinar, proponiendo escenarios que presenten la necesidad y posibilidad de trabajar de manera colectiva en interacción con varios actores, de diversa formación. Generar un espacio-taller en común a todos los cursos de ingreso, donde futuros estudiantes de las distintas carreras puedan trabajar en equipo, conocerse, interactuar y superar la fragmentación disciplinar para reconocer en el otro un posible "colega" para la acción social, alejando los modelos de competencia y rivalidad (González Valdés, 2001).

- Generar acciones para la formación de ciudadanos con mirada crítica, construyendo el COMPROMISO SOCIAL desde un rol protagónico de la Universidad, y que podría (y debería) naturalizarse en las funciones universitarias futuras (Gasca-Pliego y Olvera-García, 2011). Involucrar en la construcción de conocimientos a estudiantes informados, responsables y comprometidos que puedan tomar conciencia respecto de su potencial de transformación sobre las problemáticas sociales, tanto como a los organismos existentes, a nivel institucional y social, y sus actividades, colaborando así con la creación de una CIUDADANÍA SOCIAL, concepto que plantea el ejercicio de los derechos con plenitud por parte de los ciudadanos (Gasca-Pliego y Olvera-García, 2011).

- Retomar microescenarios de la realidad para la propuesta de trabajo, fieles a su multiactoralidad, diversidad y especial complejidad para la interacción, en contraposición a representar un escenario teórico-disciplinario abstracto, codificado en exclusiva para sus "afiliados" (Casco, 2007:2) y escindido del contexto en que se desarrolla, fenómeno descrito por Schwab (1983, como se citó en Davini, 2008:68) como "fugas teóricas".

En contraposición al esquema que usualmente enfrenta a los estudiantes a la resolución de problemas con una única respuesta correcta, circunscriptos en las propias disciplinas e incluso en cada asignatura de manera aislada, en el espacio que proponemos se los invitaría a trabajar, como los presenta González Valdés (2001:10), sobre los verdaderos "problemas mal estructurados" de la realidad, donde es crucial el trabajo colaborativo, interdisciplinar e intercultural debido a la complejidad, dinamismo y multicausalidad de aquellos.

Entrelazando las tres premisas, ideamos un espacio pasible de ser incorporado en el currículum de cualquier carrera universitaria, encua- 
drado en aprendizaje basado en problemas (ABP), donde los estudiantes se enfrentan al problema antes de un determinado aprendizaje: es decir, el problema guía al aprendizaje. Los estudiantes tienen que descubrir qué es lo que necesitan aprender, lo que incrementa notablemente la motivación al saber por qué están aprendiendo un nuevo conocimiento. No sólo se busca fomentar la enseñanza del pensamiento crítico como herramienta para detectar y comprender los problemas del mundo real (Sternberg, como se citó en Diaz Barriga, 2005:87), los cuales no están «bien» estructurados, sino promover nuevas experiencias/estrategias que logren una "motivación intrínseca procesal, motivo centrado en el proceso, en la tarea"1 (González Valdés, 2001:36). Siguiendo con los conceptos de Sternberg y Lubart (1997, como se citó en González Valdés, 2001:10), "Para tener éxito en la carrera escogida por ellos, los estudiantes necesitan practicar con problemas mal estructurados, que reflejan la vida más allá del salón de clases. Esta habilidad es crucial."

Desde el enfoque social de la problematización, según lo plantea González Valdés (2001:33), esto se encuadra también en la gran importancia de la "dimensión afectiva"2 en el desarrollo de esta competencia. El aprendizaje en el contexto de necesidad-de-resolver-un-problema tiende a almacenar o guardar el conocimiento en patrones de memoria que facilitan su posterior recuperación para resolver problemas iguales, semejantes, o aún con diferencias, pero que se resuelven por transferencia. Entre los aspectos afectivos de mayor relevancia se destacan: el rol del instructor en incentivar el 'se puede hacer' y limitar el desaliento; y la estimulación del compañerismo en una obra común.

\section{(DES)ESTRUCTURACIÓN DEL NUEVO ESPACIO DE APRENDIZAJE}

Rompiendo con la estructura que parece enmarcar todas nuestras acciones en el ámbito académico, el espacio propuesto, al que referiremos de aquí en adelante como Taller, se organizaría en los siguientes momentos:

\section{Momento 0: "Introducción"}

En un primer encuentro, se presentaría el Consejo Social, sus ob-

\footnotetext{
1 -"A la luz del carácter social de la problematización y de la importancia de analizar su dimensión afectiva (...) se analiza un experimento cubano de problematización aplicada a la creatividad en la industria. (...) Los resultados obtenidos en cuanto a la motivación resultan de interés por cuanto arrojan luz acerca de un tipo de motivación muy característica de la creatividad: la motivación procesal intrínseca. La motivación intrínseca procesal tiene la peculiaridad de producir satisfacción y placer intensos durante la realización de la tarea, aún sin llegar a obtener los resultados. Su gran poder de sostén permite avanzar de un objetivo parcial a otro, algunos de ellos poco nítidos para la persona y energiza al individuo hasta llegar al resultado final. Sin bien los participantes comenzaron a actuar básicamente por un tipo de motivo de solución de problemas (motivo centrado en la meta), gradualmente se desarrolló en ellos la motivación intrínseca procesal (motivo centrado en el proceso, en la tarea)." González Valdés, 2001: 35.

2 Sobre el "ABP + aprendizaje cooperativo o cooperado (Universidad de McMaster de Ontario, Canadá): estudios han demostrado que refuerza la dimensión afectiva de la problematización, en una mayor seguridad en la elaboración de metas, en la elaboración y recepción de feedback desde o hacia otros, el reconocimiento de la fortaleza de los demás sin detrimento de la propia autoestima y respuestas positivas a metas con alto reto e incertidumbre."Op. Cit., p. 33.
} 
jetivos y los ejes de trabajo; funcionando a su vez como un canal de difusión de este Consejo y de su agenda, presentándose desde los primeros pasos de los estudiantes en su trayecto universitario. Se organizaría un espacio de debate donde se pudiera generar conciencia sobre la visión del compromiso social por parte de la Universidad pública.

Se armaría una comisión por cada eje de la agenda comunitaria. Los estudiantes podrían elegir en qué comisión participar según sus intereses personales, buscando así fomentar la motivación y participación activa, motores principales del aprendizaje.

\section{Momento 1: "Estado inicial"}

En cada comisión, se expondrían los temas/demandas que hubieran llegado a tratarse en los plenarios del CS, los cuales nuclean la voz de muchos actores sociales e instituciones de representación, ya sea que se encuentre formando parte o no de la agenda comunitaria.

La actividad central del taller, se iniciaría con la elección de uno de aquellos temas para ser luego abordado en grupo, el cual se formaría de manera interdisciplinar, independientemente de la facultad en la cual se hubieran inscripto y únicamente dependiente de las necesidades de la demanda sobre la cual trabajarán. En el marco del ABP, cada estudiante tendría el desafío de averiguar qué necesita aprender para abordar la problematización de la demanda desde el perfil de la carrera en la cual se inscribió, poniendo en juego las incumbencias que creen que tendrán como profesionales, sus límites y la necesidad de la articulación con otros campos.

A cada grupo de estudiantes se asignarían actores de ámbitos académicos y extra-académicos cumpliendo la función de tutores, de acuerdo a la necesidad y al contexto de las demandas.

\section{Momento 2: "Búsqueda"}

En esta etapa, se buscará el pasaje de una motivación centrada en la meta inicial a una motivación centrada en el proceso (González Valdéz, 2001), donde se configuraría un espacio interactoral de diálogo atravesado por las operaciones que llevarían, o no, hacia el planteo de la/s solución/es de la demanda. Este espacio dinámico, se organizaría a partir de acciones modeladas por el contexto y conocimientos de los diversos actores (González Valdéz, 2001), donde la realidad podría imponer sus propias limitaciones. Dentro de aquellos conocimientos, se pondrían en juego los saberes previos de los estudiantes (sociales, culturales) que a través del diálogo con los saberes académicos y de la naturaleza del problema, representados en las tutorías, podrían ser valorizados o reformulados.

En este proceso se trabajaría en la problematización del qué, cómo,

3 Boles (1990) define la búsqueda como el conjunto de acciones mediante el cual se obtiene el estado final deseado. (A. González Valdés, 2001). 
con quiénes, para qué, de manera de ir planteando un "mapa" del problema e identificando actores posibles que se involucren directa o indirectamente, en dirección hacia la reducción progresiva del espacio del problema (González Valdéz, 2001).

Se podría considerar la posibilidad de un encuentro en el campo de la demanda/problemática, el cual derivaría en nuevas propuestas de acciones o necesidad de participación de otros actores en base a dicho acercamiento, siendo parte del espacio dinámico del proceso y reconociendo en él la naturaleza iterativa de la búsqueda de soluciones.

Se podría considerar la posibilidad de un encuentro en el campo de la demanda/problemática, el cual derivaría en nuevas propuestas de acciones o necesidad de participación de otros actores en base a dicho acercamiento, siendo parte del espacio dinámico del proceso y reconociendo en él la naturaleza iterativa de la búsqueda de soluciones.

\section{Momento 3: "Estado final/Final abierto"}

El "mapa" del problema, intentaría convertirse en una "carta de navegación", en el planteo de una estrategia. El "final abierto" implica reconocer las motivaciones del proceso que habrían derivado en la toma de ciertas decisiones, y considerar que en la "meta" planteada existiría la posibilidad de nuevas problematizaciones. Comprender esta dinámica como el devenir de la vida en sociedad y valorarla como el motor motivacional de nuevas búsquedas de respuesta. Cada grupo presentaría en plenario sus reflexiones sobre la experiencia. Se realizaría la puesta en común de los grupos dentro de cada comisión, con el consiguiente cierre del Taller.

Por último, imaginamos un contraste con aquella situación inicial desde la cual los imaginarios o supuestos acerca de las capacidades individuales excluyentes o incumbencias exclusivas de cada campo disciplinar, podría redefinirse y orientarse hacia una visión más amplia e integradora de los saberes específicos, y contribuir con un aprendizaje cooperativo o cooperado que refuerce su vínculo de pertenencia tanto con la comunidad académica de un sistema educativo efectivamente público y democrático, como con la sociedad con la cual solidariamente interactúen, en pos de una sociedad plural y democrática. 